\title{
The proof of efficacy in psychopharmacology: clinical studies, metaanalysis and evidence-based medicine
}

\author{
Siegfried Kasper
}

Published online: 17 October 2009

(C) Springer-Verlag 2009

Evidence-based medicine (EBM) becomes more prominent in everyday clinical practice since treatment guidelines are available for different diseases and clinical situations. However, in daily clinical practice it is quite often apparent that the type of studies which we wish to have for the specific and individual situation in which the patient is situated is not available. EBM is only possible when sound studies are conducted and additionally meta-analyses help to guide us through the plethora of available data. The critical reflections of recent meta-analyses substantiated that they are not the highest level of evidence as sometimes suggested since meta-analyses can only be as good as the selected and included studies and furthermore depend on the methodology used.

These three topics, the importance of EBM, the methodology of meta-analyses as well as the principle standards and problems in proving efficacy in clinical psychopharmacology, are dealt with in this special issue by wellknown European authorities in psychopharmacology and clinical psychiatry. The literature on the above mentioned topics is discussed and reflected critically including the possibilities, problems and limitations. It is apparent that meta-analyses can be helpful in one way, but also can be problematic when certain standards are not set and met sufficiently.

This special issue should be a guidance not only for researchers and clinicians, but also for health regulatory authorities who have to judge the available studies on clinical efficacy as well as meta-analyses. Furthermore, the contents of these papers are important for the judgement of treatment guidelines and how recommendations can be implemented in everyday clinical practice. 